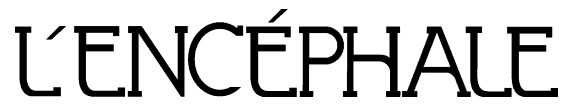

Disponible en ligne sur www.sciencedirect.com

\title{
ScienceDirect
}

journal homepage: www.em-consulte.com/produit/ENCEP

MISE AU POINT

\section{Perspectives actuelles dans la microdélétion 22q11.2: prise en charge du phénotype neurocomportemental}

\section{Neurocognitive and psychiatric management of the 22q11.2 deletion syndrome}

\author{
C. Demily ${ }^{a, *, b}$, M. Rossi ${ }^{c, d}$, M. Schneider ${ }^{e}$, P. Edery ${ }^{c, d}$, \\ A. Leleu ${ }^{f}$, T. d'Amato ${ }^{a, i}$, N. Franck ${ }^{b, g}$, S. Eliez ${ }^{e, h}$
}

\footnotetext{
a Centre de dépistage et de prise en charge des troubles psychiatriques d'origine génétique, pôle Ouest, centre hospitalier Le Vinatier, 95, boulevard Pinel, 69677 Bron cedex, France

b Centre de neuroscience cognitive, UMR 5229 (CNRS et université Lyon 1), 67, boulevard Pinel, 69675 Bron cedex, France

c Service de génétique et centre de référence des anomalies du développement, GHE, hospices civils de Lyon, 59, boulevard Pinel, 69500 Bron, France

d Centre de recherche en neurosciences de Lyon, Inserm U1028, UMR CNRS 5292, université Claude-Bernard

- Lyon 1, 95, boulevard Pinel, 69500 Bron, France

e Unité de recherche de l'Office médico-pédagogique, département de psychiatrie, faculté de médecine, université de Genève, 24, rue du Général-Dufour, 1211 Genève 4, Suisse

f Université de Bourgogne, esplanade Erasme, 21078 Dijon, France

g SUR, centre hospitalier Le Vinatier, 95, boulevard Pinel, 69500 Bron, France

h Département de médecine génétique et développement, faculté de médecine, université de Genève Michel-Servet 1, 24, rue du Général-Dufour, 1211 Genève 4, Suisse

i Pôle Est, centre hospitalier Le Vinatier, 95, boulevard Pinel, 69677 Bron cedex, France
}

Reçu le 24 octobre 2013 ; accepté le 12 juin 2014

\section{MOTS CLÉS}

$22 q 11.2$;

Syndrome de

DiGeorge ;

Remédiation

cognitive ;

\begin{abstract}
Résumé La microdélétion 22q11.2 est le syndrome microdélétionnel le plus fréquent de la population générale. Le phénotype associe des anomalies de l'appareil pharyngé embryonnaire à un phénotype neurocomportemental. La présentation clinique du syndrome est extrêmement variable d'un individu à l'autre, quelle que soit la taille de la délétion, et plus de 180 manifestations ont été décrites, aucune n'étant pathognomonique. Les symptômes psychiatriques, particulièrement de nature psychotique, sont fréquents dans la microdélétion 22q11.2
\end{abstract}

* Auteur correspondant.

Adresse e-mail : caroline.demily@ch-le-vinatier.fr (C. Demily). 
ADHD ;

Psychose ;

Anomalies

cytogénétiques ;

Neurocognition ;

Cognition sociale ;

Schizophrénie

\section{KEYWORDS}

22q11.2 deletion

syndrome;

DiGeorge's syndrome;

Chromosomal

abnormalities;

Psychosis;

Cognitive

remediation;

ADHD;

Neurocognition;

Social cognition;

Schizophrenia et de nombreux psychiatres sont amenés à rencontrer ces patients. La prise en charge doit tenir compte des particularités du syndrome. L'évaluation de la neurocognition, de la cognition sociale et la recherche de symptômes psychiatriques dans le contexte médical général sont une étape fondamentale. Concernant le phénotype neurocomportemental, les soins de remédiation cognitive doivent être combinés à une prescription mesurée de psychotropes pour aboutir à une meilleure qualité de vie de ces patients.

(c) L’Encéphale, Paris, 2014.

\begin{abstract}
Summary
Introduction. - The 22q11.2 deletion syndrome (22q11.2DS) is caused by hemizygous microdeletions on chromosome 22. 22q11.2DS has several presentations including Di George's syndrome, velo-cardio-facial syndrome or Shprintzen's syndrome and it is the most frequent microdeletion syndrome in the general population (prevalence estimated at 1/4000 births, de novo: $90 \%$ ). The inheritance of the syndrome $(10 \%)$ is autosomal dominant. Most people with 22q11.2DS are missing a sequence of about 3 million DNA building blocks (base pairs) on one copy of chromosome 22 in each cell. A small percentage of affected individuals have shorter deletions in the same region (contiguous gene deletion syndrome). The general features of 22q11.2DS vary widely (more than 180 phenotypic presentations) and the syndrome is under diagnosed. Characteristic symptoms may include congenital heart disease, defects in the palate, neuromuscular problems, velo-pharyngeal insufficiency, hypoparathyroidism, craniofacial features and problems with the immune system T-cell mediated response (caused by hypoplasia of the thymus).

Cognitive phenotype. - The neurocognitive phenotype of the 22q11.2DS is complex. Cognitive deficits are seen in the majority $(80-100 \%)$ of individuals with 22q11DS with impairments in sustained attention, executive function, memory and visual-spatial perception. Borderline intellectual function (IQ: $70-75$ ) is most common, mild intellectual disability (IQ: $55-75$ ) is slightly less frequent and a small percentage of children fall into the low average intelligence range. Most children with 22q11.2DS achieve higher scores in verbal tasks than in non-verbal tasks, although this pattern of dysfunction being not universal. Brain MRI studies have shown volumetric changes in multiple cortical and subcortical regions in individuals with 22q11DS that could be related to both cognition and psychoses.

Psychiatric phenotype.-General psychiatric features included anxiety disorders, attention deficit disorder and poor social skills (40-50\%). An elevated risk of bipolar disorder and major depression occurs in adolescence and young adulthood. A strong and specific relationship exists between the presence of the 22q11.2 microdeletion and schizophrenia (30-40\%). This risk is not associated with any other neurogenetic syndrome. Social cognition is impaired in $22 q 11.2$ DS and this observation is correlated with psychotic features. So, long-term medical care is increasingly being directed towards the treatment and recognition of these symptoms.

Treatment. - Required pharmacological treatment strategies have to be adapted to the syndrome. Moreover, cognitive remediation is a promising tool for treating neuro- and social cognitive deficits in 22q11.2DS. However, these new therapeutic strategies have to be developed to improve quality of life.

(c) L’Encéphale, Paris, 2014.
\end{abstract}

\section{Introduction : la prise en charge du phénotype neurocomportemental des affections cytogénétiques}

La prévalence des affections génétiques en population de sujets adultes souffrant d'un trouble mental est importante. Malheureusement, les patients qui en souffrent ne bénéficient pas tous d'un diagnostic ou d'une prise en charge spécifique en milieu psychiatrique alors que des centres de références spécialement dévolus à ces affections existent en France. Cette lacune peut s'expliquer par l'histoire même de la psychiatrie dont les acteurs de soin ont longtemps revendiqué un modèle non médical, opposant traditionnellement les soins du psyché à la médecine somatique, voire étant réfractaire à celle-ci.

De ce fait, les généticiens d'un côté et les psychiatres de l'autre n'ont que de très exceptionnels points de rencontre concernant la prise en charge des adultes.

De cette dichotomie, résultent plusieurs observations:

- le diagnostic d'une affection génétique à l'origine des symptômes psychiatriques de l'adulte n'est souvent pas fait si des points d'appel cliniques somatiques ne sont pas au premier plan;

- l'offre de soins psychiatriques spécifiques (et en particulier de réhabilitation) destinée aux patients adultes souffrant d'une affection génétique est insuffisante ; 
- les soins spécifiques mis en place pour les enfants ne sont pas facilement poursuivis à l'âge adulte.

Parmi les affections cytogénétiques qui ne sont parfois prises en charge qu'en santé mentale, la microdélétion 22q11.2 occupe une place prépondérante. En effet, parmi les patients atteints de schizophrénie, 1 à $2 \%$ d'entre eux sont porteurs d'une microdélétion 22q11.2. Chaque psychiatre a donc été amené à prendre en charge ces patients, pour lesquels une connaissance du phénotype neurocomportemental et l'adaptation des outils de soins sont primordiales. Une des complexités du phénotype réside en l'association variable d'un phénotype neurocomportemental avec des anomalies du développement général.

\section{La microdélétion 22q11.2: données générales}

Le syndrome de microdélétion 22q11.2 (22q11DS) est caractérisé par une grande hétérogénéité clinique intra- et interfamiliale [1]. Plus de 180 manifestations cliniques ont été décrites et aucune d'entre elle n'est pathognomonique, ce qui représente une difficulté importante pour le diagnostic et la prise en charge.

Une dysmorphie discrète mais caractéristique est présente incluant des oreilles petites avec des anomalies de plicature, des fentes palpébrales étroites, un hypertélorisme, un nez tubulaire, une petite bouche, un visage long, une micrognathie, des doigts longs et fins.

Une hypoacousie de transmission ou de perception doit être systématiquement écartée.

Plus de $70 \%$ des patients présentent une malformation cardiaque notamment de type conotroncale incluant la communication inter-ventriculaire (CIV) ou, plus rarement, la communication inter-atriale $(\mathrm{CIA})$, la tétralogie de Fallot, les anomalies de l'arc aortique, le tronc artériel commun. Les anomalies du palais sont également fréquentes $(69 \%$ des cas) et de gravité variable. Certains patients présentent une fente palatine, qui peut parfois s'associer à une micrognathie et une glossoptose dans le cadre d'une séquence de Pierre-Robin. D'autres patients présentent une luette bifide, une fente sous-muqueuse et une insuffisance vélaire qui, d'une part, est responsable d'une voie nasonnée et, d'autre part, peut favoriser la survenue de difficultés alimentaires (difficultés de succion/déglutition, fausses routes, régurgitations).

Une hypoplasie du thymus peut être associée et un déficit immunitaire de sévérité variable est observé dans $77 \%$ des cas. Les patients peuvent présenter des infections récurrentes de gravité variable et une baisse notamment des taux de lymphocytes $T$; une immunodéficience sévère est cependant rare. Par ailleurs, la survenue d'otites et d'infections respiratoires récurrentes peut être favorisée aussi par les anomalies palatines. Des pathologies auto-immunes peuvent également être observées, incluant des thyroïdites, des arthrites, un vitiligo, une thrombocytopénie. Rarement, un syndrome de Bernard-Soulier (macrothrombocytopénie, tendance aux saignements) peut être observé, si une mutation de la copie non délétée du gène GP1BB s'associe à la délétion de la région $22 q 11.2$.
Une hypoplasie des parathyroïdes est responsable d'une hypocalcémie observée dans environ la moitié des cas, notamment pendant les premières semaines de vie.

Plusieurs autres anomalies malformatives peuvent être observées, incluant des anomalies rénales (31\%), oculaires et, plus rarement, gastro-intestinales, du système nerveux central, du squelette et des extrémités. La scoliose est parfois observée $(15 \%)$.

L'atteinte neurologique est également très variable et peut inclure une hypotonie, un décalage des acquisitions psychomotrices, des troubles spécifiques complexes des apprentissages, une déficience intellectuelle généralement légère à modérée, des troubles du comportement et des pathologies psychiatriques : ces aspects feront l'objet d'une description détaillée dans les paragraphes suivants.

Le syndrome de Di George résulte d'une microdélétion interstitielle du bras long (q) du chromosome 22. En raison de l'expressivité phénotypique très variable, plusieurs entités syndromiques différentes avaient été décrites avant la découverte de la microdélétion. Le syndrome vélo-cardiofacial (syndrome de Shprintzen) est caractérisé par des particularités morphologiques, des anomalies palatines et cardiaques. Le syndrome de Di George ou CATCH22 (acronyme anglais pour Cardiac defects, Abnormal facies, Thymic hypoplasia, Cleft palate, and Hypocalcaemia) est défini par des phénotypes plus sévères comportant des malformations cardiaques, une hypocalcémie, un déficit immunitaire, une dysmorphie, des anomalies palatines et d'autres possibles anomalies du développement. La microdélétion 22q11.2 a été identifiée comme la seule entité responsable de ces différentes manifestations cliniques [2,3]: la terminologie syndrome de microdélétion 22q11 (22q11DS) est donc actuellement retenue comme la plus adaptée.

La prévalence supposée du syndrome est de 1 naissance sur 4000 à 6000 (probablement sous-estimée en raison de l'existence de phénotypes atténués).

La microdélétion survient de novo dans $90 \%$ des cas [4] et est héritée dans $10 \%$ des cas de l'un des deux parents, qui est alors souvent pauci-symptomatique. Une personne atteinte a une probabilité de $50 \%$ à chaque conception de transmettre la microdélétion à sa descendance.

La plupart des patients (85-90\%) présentent une délétion d'environ $3 \mathrm{Mb}$ incluant une trentaine de gènes, alors qu'une minorité d'entre eux présentent des délétions plus courtes [1]. L'expressivité clinique très variable du syndrome n'est pas corrélée avec la taille de la délétion, ce qui amène la notion de syndrome de gènes contigus [5]: les facteurs de modification de la sévérité phénotypique sont actuellement inconnus.

Le diagnostic est généralement suspecté sur le plan clinique et confirmé par une analyse d'hybridation in situ fluorescente (FISH) ; d'autres techniques peuvent être également utilisées comme l'Analyse chromosomique sur puce à $A D N(A C P A, 1)$.

La prise en charge médicale des patients est complexe et multidisciplinaire et nécessite une coordination optimale parmi les différents intervenants [1]; elle dépend des symptômes et peut inclure un suivi pédiatrique, généraliste, cardiologique, ORL, psychiatrique, génétique, neuropédiatrique, immunologique, odontologique, gastroentérologique, ophtalmologique, orthopédique et néphro-urologique, une prise en charge socio-éducative et 
des rééducations (psychomotricité, orthophonie et ergothérapie).

Parmi les facteurs génétiques de risque de développement d'une psychose, la microdélétion $22 q 11.2$ est le plus fréquent (25-35\% des cas). L'étude du phénotype neurocognitif chez les patients, pourrait permettre de mieux reconnaître les symptômes prédictifs ou précurseurs de l'émergence des troubles psychotiques et donc d'y remédier précocement par une prise en charge adaptée.

\section{Le phénotype neurocognitif et les anomalies de la cognition sociale}

\section{Les troubles neurocognitifs}

Dans de nombreuses affections cytogénétiques comme la microdélétion 22q11.2, des troubles neurocognitifs sont intriqués aux symptômes psychiatriques. Les difficultés d'apprentissage scolaire sont fréquentes dans le 22q11.2DS et peuvent être expliquées, dans plus d'un tiers des cas, par une déficience intellectuelle légère (QI total $<70$ avec QI verbal > Q performance) [6]. La description des troubles neurocognitifs dans le 22q11.2DS est une tâche complexe car le profil des patients n'est pas homogène et n'est pas stable dans le temps. Le déficit neurocognitif dans la microdélétion 22q11.2 serait plus important dans la forme héritée que dans la forme de novo mais cette hypothèse reste à confirmer et pourrait prédominer chez les sujets de sexe masculin mais les résultats sont inconstants [7].

L'hypothèse neurodéveloppementale la plus probable serait une perturbation précoce des processus de traitement de l'information spatiale et temporelle, ce qui induirait des anomalies dans les processus cognitifs visuo-spatiaux. Les difficultés visuo-spatiales seraient prédictives de l'échec à appréhender les mathématiques [8] rendant compte des difficultés scolaires s'installant classiquement en milieu de primaire. En effet, l'apprentissage de l'arithmétique fait appel au dénombrement et à la comparaison, mécanismes basés sur la représentation spatiale des quantités $[9,10]$. Les difficultés d'intégration visuo-motrices couplées à la dyspraxie compliqueraient considérablement toutes les tâches papier-crayon avec représentation spatiale (comme la géométrie, par exemple). Les troubles exécutifs (résolution de problèmes et planification) sont aussi un aspect important du dysfonctionnement cognitif. L'existence fréquente d'un déficit attentionnel (touchant surtout l'attention visuo-spatiale) pourrait aussi affecter les capacités de planification de l'enfant. Les troubles de l'attention soutenue sont également bien décrits [11]; une étude a rapporté, chez les patients à haut risque de développement ultérieur d'une psychose, un profil cognitif proche de celui observé dans la schizophrénie [12]. Les fonctions langagières sont également perturbées dans le sens d'un retard des acquisitions, accessible à l'orthophonie. Par ailleurs, l'insuffisance vélaire et la rhinolalie qui en sont les conséquences ont un impact négatif sur le développement du langage (altérations phonétiques).

Le fonctionnement cognitif est la seule variable ayant un impact sur la dextérité des patients, le sexe ou l'existence d'une cardiopathie congénitale n'ayant pas d'influence significative sur la motricité [13].
Le déficit neurocognitif semble le plus souvent inhérent à la microdélétion $22 q 11.2$ et non au phénotype psychiatrique de survenue différée [14], bien qu'un affaiblissement du QI verbal puisse agir comme facteur prédisposant aux troubles psychotiques chez l'adolescent. À l'âge adulte, l'existence de formes neurodégénératives avec altérations progressives des capacités cognitives, retrait et apragmatisme sévères (5 à 10\%), complique la prise en charge. Ces formes sont peu accessibles au traitement et aboutissent souvent à l'institutionnalisation des patients. Leur physiopathologie exacte reste peu connue $[15,16]$ mais le profil neurocognitif retrouvé ne correspond pas tout à fait au profil des démences de type Alzheimer, bien que la région $22 q 11.2$ soit une région de susceptibilité [17].

La microdélétion 22q11.2 est associée à un volume cérébral réduit surtout dans la partie postérieure du cortex $[18,19]$. La réduction de volume atteindrait surtout la substance blanche et dans une moindre mesure la substance grise [18]. Les réseaux fronto-striataux et fronto-pariétaux seraient plus spécifiquement impliqués dans les dysfonctionnements cognitifs et dans la gestion des émotions [19].

Les études portant sur le phénotype neurocognitif et comportemental dans la microdélétion 22q11.2 ont des limites importantes: faibles cohortes, aspects longitudinaux, impact sélectif des troubles attentionnels, liens avec les troubles de la cognition sociale encore peu étudiés... La genèse des troubles psychotiques dans ce contexte si particulier n'est pas encore élucidée mais il pourrait s'agir d'un formidable challenge pour comprendre le rôle des troubles du traitement de l'information dans l'émergence des troubles mentaux, non seulement dans la microdélétion 22q11.2 mais également dans la population générale.

\section{Les troubles de la cognition sociale}

Les patients porteurs de la microdélétion 22q11.2, particulièrement lorsque des symptômes psychotiques sont associés, présentent un déficit important dans les échanges sociaux (repli à domicile, pauvreté des liens...). Sachant qu'un lien existe entre symptomatologie et reconnaissance des émotions faciales chez le patient souffrant de schizophrénie [20], il est envisageable que les déficits observés chez les patients souffrant d'une microdélétion $22 q 11.2$ soient de même nature. Bien que plus récemment mise en évidence et moins largement évaluée, la cognition sociale est une composante essentielle des dysfonctionnements cognitifs observés chez les patients souffrant de microdélétion 22q11.2 [21]. Elle englobe toute activité mentale de traitement de l'information sociale dans le but de reconnaître et comprendre les états mentaux d'autrui, notamment l'attribution des intentions, l'empathie ou encore la reconnaissance des émotions faciales. Les troubles de la cognition sociale sont associés au développement des symptômes psychotiques chez les patients atteints de microdélétion 22q11.2 [22]. L'impact des troubles de la cognition sociale sur les interactions entretenues avec autrui constitue sans nul doute l'une des causes principales du retrait social et des difficultés d'adaptation dont peuvent souffrir les patients.

Plusieurs études rapportent un déficit de reconnaissance des émotions faciales chez le patient souffrant de 
microdélétion 22q11.2 [11,22-24]. De manière comparative à des patients porteurs du syndrome de Williams (qui s'accompagne de troubles neurocognitifs et d'une hypersociabilité), les patients porteurs de la microdélétion 22q11.2 reconnaissent mal les émotions d'autrui et se représentent difficilement les états mentaux de l'autre, et ce d'autant que des symptômes psychotiques sont associés [25]. Ce déficit pourrait prédominer sur la reconnaissance de la peur, de la colère et du dégoût alors que la reconnaissance de la joie et de la surprise serait plus efficiente [11].

Par exemple, alors que la région des yeux est très informative pour des participants sains à qui des visages expressifs sont présentés, les patients l'explorent beaucoup moins [11]. En outre, un lien entre ces difficultés et la détérioration des habiletés visuo-spatiales et/ou le risque de développer un trouble psychotique a été suggéré [22]. Une atteinte de la flexibilité cognitive pourrait aussi être responsable de ce déficit car les patients manquent d'adaptabilité lors des tâches de catégorisation [26].

Cependant, les aspects altérés de la cognition sociale dans la microdélétion 22q11.2 diffèrent de ceux classiquement rencontrés dans l'autisme [26].

Les troubles de la cognition sociale sont une piste intéressante pour comprendre la transition psychotique dans le 22q11DS [21]. Cependant, les aspects neurodéveloppementaux de ce déficit ainsi que les liens avec les dysfonctionnements neurocognitifs ne sont pas encore clairement élucidés.

\section{Le phénotype psychiatrique}

Le phénotype psychiatrique associé à la microdélétion 22 q11.2 a, dès les années 1990, reçu une large attention de la part de la communauté internationale. En effet, l'association d'un risque de trouble psychotique augmenté de 25 à 30 fois a fait de cette maladie rare un modèle génétiquement homogène pour l'étude et la compréhension du développement de la psychose [27].

$\mathrm{Au}$ cours des 20 dernières années, aussi bien les symptômes psychotiques que les déficits cognitifs ou la clinique associés à la psychose dans la microdélétion 22 q11 ont été largement étudiés. Selon les études, les symptômes psychotiques affectent environ $60 \%$ des adolescents et adultes concernés [27-29]. Le diagnostic formel de schizophrénie ou de trouble schizo-affectif est lui posé chez 5 à $10 \%$ des adolescents [28,30] et 25 à $40 \%$ des adultes [6,31]. Le tableau clinique, même s'il survient chez des individus qui, typiquement, ont une diminution des compétences intellectuelles plus marquée que la population des patients schizophrènes en général, constitue un tableau qui ne présente pas de différence majeure avec celui de la psychose en général [32].

Au-delà des troubles psychotiques, un certain nombre de difficultés psychiques apparaissent fréquemment dans le contexte de la microdélétion 22q11. Même si ces dernières sont moins spécifiques à cette population, elles méritent une attention particulière, en raison des retentissements possibles de ces troubles sur le fonctionnement quotidien. En particulier, les troubles anxieux sont fréquemment observés, aussi bien durant l'enfance ou l'adolescence que durant l'âge adulte [33-35]. Ces derniers ont reçu une attention particulière au cours des dernières années, en raison de leur association avec l'émergence de symptômes ou de troubles psychotiques. En effet, une récente étude longitudinale de Gothelf et al. [30] a montré que la présence d'un trouble anxieux était un prédicteur particulièrement fort du développement d'un trouble psychotique plus tard dans la vie. Dans cette étude, 9 individus sur 10 ayant développé un trouble psychotique sur une période de quatre ans présentaient un trouble anxieux lors de l'évaluation initiale. Les troubles anxieux les plus fréquemment rencontrés chez les enfants et les jeunes adolescents se caractérisent généralement par des phobies isolées, des angoisses de séparation, ou une anxiété généralisée [31,33]. À l'adolescence, les phobies sociales ou une forte anxiété sociale sont souvent présentes, alors que les attaques de panique apparaissent plutôt à l'âge adulte.

Les troubles affectifs, notamment les épisodes dépressifs isolés ou répétés ainsi que la dysthymie, tendent à émerger dans la seconde partie de l'adolescence et chez le jeune adulte $[30,31]$. Ils ont été mis en rapport tant avec les difficultés d'adaptation dues aux particularités physiques et intellectuelles du syndrome qu'à une altération à proprement parler du système limbique et des structures cérébrales impliquées dans la régulation de l'humeur.

Les déficits d'attention sont une des difficultés les plus fréquemment rencontrée par les personnes avec une microdélétion 22q11, tant durant l'enfance et l'adolescence qu'à l'âge adulte $[31,36]$. Les difficultés d'attention, généralement sans hyperactivité associée, se caractérisent généralement par des oublis nombreux, une tendance à passer d'une activité non terminée à une autre ou forte distractibilité. Un trouble de l'attention sans hyperactivité associée est généralement plus difficile à repérer qu'un trouble de l'attention avec hyperactivité. Cependant, les déficits d'attention ont un retentissement sur les apprentissages et doivent donc être soigneusement diagnostiqués.

Au cours des dernières années, des publications ont suggéré une augmentation des troubles du spectre autistique dans cette population [37]. Il reste aujourd'hui encore à clarifier si cette symptomatologie n'est pas en partie attribuable à une difficulté du fonctionnement social, qui tend à se marquer plutôt à partir du développement pubère, et qui peut être, chez certains adolescents matures ou jeunes adultes, une source de handicap en tant que tel.

\section{Stratégies de prises en charge}

\section{La thérapeutique médicamenteuse}

L'entrée dans la psychose semble survenir plus précocement, en moyenne autour de 18 ans. S'il existe encore relativement peu d'études concernant le traitement de la psychose dans le contexte de la microdélétion 22, il semble que ces patients répondent aux antipsychotiques de seconde génération. La sévérité du trouble psychotique survenant dans ce contexte nécessite parfois une combinaison d'antipsychotiques pour maîtriser efficacement les symptômes. Dans notre expérience, il convient de traiter les symptômes psychotiques lorsqu'ils deviennent cliniquement envahissants ou lorsqu'ils apparaissent plusieurs fois par mois. 
De plus, la fréquente association d'un trouble affectif, conduisant au diagnostic de trouble schizo-affectif, amène également, dans environ un tiers des cas, à la nécessité de combiner une médication antidépressive, ou de thymorégulation, avec la médication antipsychotique.

Certaines particularités métaboliques du syndrome (notamment l'haplo-insuffisance du gène COMT, impliqué dans le métabolisme des catécholamines et notamment de la dopamine) ont conduit à des propositions de traitement expérimental, permettant de mieux cibler l'origine des symptômes [38,39]. Ces publications restent aujourd'hui trop anecdotiques pour permettre de réelles conclusions.

Finalement, les suppléments d'oméga 3/oméga 6 représentent une piste intéressante pour la réduction des symptômes psychotiques dans cette population. En effet, même s'il n'y a pas de littérature spécifique sur ce sujet dans la microdélétion $22 q 11$, il est possible de faire l'analogie avec des études réalisées auprès de jeunes patients ayant un risque de psychose [40].

Comme indiqué dans le chapitre précédent, la gestion des troubles anxieux chez les adolescents avec une microdélétion 22q11 est devenue l'un des focus thérapeutiques principaux, en raison de leur association avec le développement ultérieur de troubles psychotiques. À notre connaissance, il n'existe pas d'essai clinique évaluant la prise en charge des troubles anxieux dans la microdélétion 22q11. Il convient donc de suivre les recommandations thérapeutiques pour les troubles anxieux dans les populations pédiatriques, à savoir une prise en charge multimodale associant psychothérapie et traitement psychopharmacologique (inhibiteurs de recapture de la sérotonine).

À l'heure actuelle, le traitement des troubles de l'attention est le mieux documenté dans la littérature sur la microdélétion $22 q 11$. Les recherches suggèrent que les enfants et les adolescents avec une microdélétion 22q11 répondent favorablement à des traitements de psychostimulants [41,42]. Dans les études effectuées, le traitement par psychostimulants n'a pas été mis en lien avec une augmentation du risque psychotique, ni avec une augmentation des problèmes cardiaques. Cependant, comme ces études portent sur un nombre limité de patients, il reste impossible de tirer des conclusions définitives.

\section{La remédiation cognitive}

La remédiation cognitive permet de diminuer l'impact des troubles cognitifs (en particulier visuo-spatiaux et exécutifs) décrits plus haut. Elle vise la restauration des processus altérés ou la compensation des déficits. L'approche restauratrice vise l'entraînement direct de fonctions potentiellement altérées, alors que l'approche par compensation implique l'acquisition de nouvelles stratégies de traitement de l'information.

L'acquisition de nouvelles stratégies répond à des besoins tangibles du patient. Il faut l'amener à traiter les situations présentées en séance, afin qu'il puisse ultérieurement procéder de manière proche dans les situations de sa vie courante. Le thérapeute le conduit systématiquement à prendre conscience des stratégies qu'il met en œuvre spontanément ou qu'il développe grâce à son étayage.
Certains programmes informatisés de stimulation cognitive ont été proposés dans l'hyperactivité avec déficit attentionnel et le gain sur la mémoire de travail est stable à 6 mois [43], même chez les jeunes patients nés prématurés avec un QI total < 80 [44].

Cependant et à notre connaissance, aucune publication ne fait état de l'efficacité de la remédiation cognitive dans la microdélétion 22q11 (pratiquée dans le sens du développement de stratégies alternatives et non dans le sens d'un apprentissage par essai/erreur). Dans notre expérience de l'utilisation du programme CRT (Cognitive Remediation Therapy) chez les patients porteurs de la microdélétion [45], le développement de stratégies alternatives avec l'aide d'un thérapeute formé pourrait s'avérer extrêmement pertinent avec un transfert optimal de l'utilisation de ces stratégies dans la vie quotidienne.

Plusieurs études ont souligné l'excellente faisabilité de l'entraînement cognitif dans la microdélétion 22q11 et la facilité de son emploi à domicile. Harrell et al. [46] ont décrit le protocole CCRP qui s'effectue sur 12 semaines, le participant étant invité à s'y consacrer 4 fois par semaine ( 3 jeux par session) sur une durée totale d'approximativement 32 heures. Neuf patients sur 13 ont adhéré à l'ensemble du programme avec un indice de satisfaction important même si les effets sur la cognition sont restés modérés, ce qui souligne l'efficacité incomplète des programmes d'entraînement. Cette étude encourageante souffre également d'une limite importante : la non-inclusion des sujets atteints de psychose, cible habituellement privilégiée de la remédiation cognitive.

Le programme Vis-à-Vis est centré sur les troubles de la cognition sociale [47]. Dix enfants entraînés sur 12 semaines (20 minutes par semaine à domicile) ont vu leurs capacités à reconnaître les émotions faciales progresser. Ce programme a également eu un impact positif sur le raisonnement non verbal.

Dans les années à venir, la remédiation cognitive aura à s'adapter le plus finement possible aux difficultés présentées par les patients. C'est un point fondamental de la prise en charge qu'il faut valoriser et développer en France. En effet, un environnement favorable pourrait avoir un impact significatif sur le fonctionnement général et le comportement des patients porteurs de la microdélétion (versus population générale).

\section{Conclusion}

La création des centres de référence labellisés dans les suites du plan national-maladies rares 2005-2008 en France a permis une avancée considérable dans le dépistage et la prise en charge des affections génétiques. Les anomalies physiques, mentales, sensorielles et les troubles du comportement font partie des points d'appel cliniques. L'accès au diagnostic ainsi que la prise en charge ont été structurés. Les pédiatres, les médecins généralistes ainsi que les spécialistes de médecine organique orientent leurs jeunes patients vers ce dispositif. Les enfants dépistés, et pour lesquels un diagnostic est posé, peuvent être stimulés dès le plus jeune âge et pris en charge dans leur quotidien et notamment dans l'adaptation à la vie scolaire (orthophonie, psychomotricité, ergothérapie, auxiliaire de vie scolaire...). 
Malheureusement, la question de la transition vers l'âge adulte reste entière car les prises en charge précoces de stimulation (menées surtout chez l'enfant en orthophonie et en psychomotricité ou en ergothérapie) n'ont pas de continuité claire chez l'adulte. Il est désormais important de structurer les soins pour pouvoir remédier à cette lacune: les patients porteurs d'une affection microdélétionnelle, en particulier la microdélétion 22q11.2, ont un profil syndromique et cognitif particulier, qu'il faut évaluer et intégrer dans le contexte clinique pour pouvoir apporter des soins adaptés en psychiatrie.

\section{Déclaration d'intérêts}

Les auteurs déclarent ne pas avoir de conflits d'intérêts en relation avec cet article.

\section{Références}

[1] MCDonald-McGinn DM, Emanuel BS, Zackai EH. 22q11.2 deletion syndrome. 1999 September 23 [updated 2013 February 28]. In: Pagon RA, Adam MP, Bird TD, Dolan CR, Fong CT, Stephens K, editors. GeneReviews ${ }^{T M}$ [Internet]. Seattle (WA): University of Washington, Seattle; 1993-2013. http://www.ncbi.nlm.nih.gov/books/NBK1523/

[2] Driscoll DA, Spinner NB, Budarf ML, et al. Deletions and microdeletions of 22q11.2 in velo-cardio-facial syndrome. Am J Med Genet 1992;44:261-8.

[3] Driscoll DA, Budarf ML, Emanuel BS, et al. A genetic etiology for Di George syndrome: consistent deletions and microdeletions of 22q11. Am J Hum Genet 1992;50:924-33.

[4] Delio M, Guo T, McDonald-McGinn DM, et al. Enhanced maternal origin of the 22q11.2 deletion in velocardiofacial and Di George syndromes. Am J Hum Genet 2013;92:439-47.

[5] Sandrin-Garcia P, Abramides DV, Martelli LR, et al. Typical phenotypic spectrum of velocardiofacial syndrome occurs independently of deletion size in chromosome 22q11.2. Mol Cell Biochem 2007;303:9-17.

[6] Murphy KC, Jones LAJ, Owen MJ. High rates of schizophrenia in adults with velo-cardio-facial syndrome. Arch Gen Psychiatry 1999;56:940-5.

[7] De Smedt B, Devriendt K, Fryns JP, et al. Intellectual abilities in a large sample of children with velo-cardio-facial syndrome: an update. J Intellect Disabil Res 2007;51:666-70.

[8] Henry JC, van Amelsvoort T, Morris RG, et al. An investigation of the neuropsychological profile in adults with velo-cardio-facial syndrome (VCFS). Neuropsychologia 2002;40:471-8.

[9] Simon TJ. A new account of the neurocognitive foundations of impairments in space, time and number processing in children with chromosome 22q11.2 deletion syndrome. Dev Disabil Res Rev 2008;14:52-8.

[10] Debbané M, Glaser B, Gex-Fabry M, et al. Temporal perception in velocardiofacial syndrome. Neuropsychologia 2005;43:1754-62.

[11] Campbell L, McCabe K, Leadbeater K, et al. Visual scanning of faces in 22q11.2 deletion syndrome: attention to the mouth or the eyes? Psychiatry Res 2010;177:211-5.

[12] Lewandowski KE, Shashi V, Berry PM, et al. Schizophrenic-like neurocognitive deficits in children and adolescents with 22q11 deletion syndrome. Am J Med Genet B 2007;144:27-36.

[13] Van Aken K, De Smedt B, Van Roie A, et al. Motor development in school-aged children with 22q11 deletion (velocardiofacial/Di George syndrome). Dev Med Child Neurol 2007;49:210-3.
[14] Niklasson L, Gillberg C. The neuropsychology of 22q11 deletion syndrome. A neuropsychiatric study of 100 individuals. Res Dev Disabil 2010;31:185-94.

[15] Evers LJ, De Die-Smulders CE, Smeets EE, et al. The velo-cardio-facial syndrome: the spectrum of psychiatric problems and cognitive deterioration at adult age. Genet Couns 2009;20:307-15.

[16] Evers LJ, Vermaak MP, Engelen JJ, et al. The velocardiofacial in older age: dementia and autistic features. Genet Couns 2006; 17:333-40.

[17] Lee JH, Cheng R, Graff-Radford N, et al. Analyses of the National Institute on Aging-Late Onset Alzheimer's Disease Family Study: implication for additional loci. Arch Neurol 2008;65:1518-26.

[18] Campbell L. Brain, behaviour and cognition in 22q11.2 deletion syndrome (22q11.2DS). London: University of London; King's College London; 2006.

[19] Eliez S, Schmitt J, White C, et al. Children and adolescent with velo-cardio-facial syndrome: a volumetric MRI study. Am J Psychiatry 2000;157:409-15.

[20] Martin F, Baudouin JY, Tiberghien G, et al. Processing emotional expression and facial identity in schizophrenia. Psychiatry Res 2005; 134:43-53.

[21] Jalbrzikowski M, Carter C, Senturk D, et al. Social cognition in 22q11.2 microdeletion syndrome: relevance to psychosis? Schizophr Res 2012;142:99-107.

[22] Andersson F, Glaser B, Spiridon M, et al. Impaired activation of face processing networks revealed by functional magnetic resonance imaging in 22q11.2 deletion syndrome. Biol Psychiatry 2008;63:49-57.

[23] Glaser B, Schaer M, Berney S, et al. Structural changes to the fusiform gyrus: a cerebral marker for social impairments in 22q11.2 deletion syndrome? Schizophr Res 2007;96:82-6.

[24] Van Amelsvoort T, Schmitz N, Daly E, et al. Processing facial emotions in adults with velo-cardio-facial syndrome: functional magnetic resonance imaging. Br J Psychiatry 2006;189:560-1.

[25] Chow EWC, Watson M, Young DA, et al. Neurocognitive profile in 22q11 deletion syndrome and schizophrenia. Schizophr Res 2006;87:270-8.

[26] McCabe KL, Melville JL, Rich D, et al. Divergent patterns of social cognition performance in autism and 22q11.2 deletion syndrome (22q11DS). J Autism Dev Disord 2013;43: 1926-34.

[27] Bassett AS, Chow EW. 22q11 deletion syndrome: a genetic subtype of schizophrenia. Biol Psychiatry 1999;46:882-91.

[28] Schneider M, Schaer M, Mutlu AK, et al. Clinical and cognitive risk factors for psychotic symptoms in 22q11.2 deletion syndrome: a transversal and longitudinal approach. Eur Child Adolesc Psychiatry 2014;23:425-36.

[29] Debbané M, Glaser B, David MK, et al. Psychotic symptoms in children and adolescents with 22q11.2 deletion syndrome: neuropsychological and behavioral implications. Schizophr Res 2006;84:187-93.

[30] Gothelf D, Schneider M, Green T, et al. Risk factors and the evolution of psychosis in 22q11.2 deletion syndrome: a longitudinal two-site study. J Am Acad Child Adolesc Psychiatry 2013;52:1192-203.

[31] Green T, Gothelf D, Glaser B, et al. Psychiatric disorders and intellectual functioning throughout development in velocardiofacial (22q11.2 deletion) syndrome. J Am Acad Child Adolesc Psychiatry 2009;48:1060-8.

[32] Bassett AS, Chow EWC, AbdelMalik P, et al. The schizophrenia phenotype in 22q11 deletion syndrome. Am J Psychiatry 2003;160:1580-6.

[33] Fabbro A, Rizzi E, Schneider M, et al. Depression and anxiety disorders in children and adolescents with velocardio-facial syndrome (VCFS). Eur Child Adolesc Psychiatry 2012;21:379-85. 
[34] Jolin EM, Weller RA, Weller EB. Occurrence of affective disorders compared to other psychiatric disorders in children and adolescents with 22q11.2 deletion syndrome. J Affect Disord 2012;136:222-8.

[35] Fung WLA, McEvilly R, Fong J, et al. Elevated prevalence of generalized anxiety disorder in adults with 22q11.2 deletion syndrome. Am J Psychiatry 2010;167:998.

[36] Antshel KM, Hendricks K, Shprintzen R, et al. The longitudinal course of attention deficit/hyperactivity disorder in velo-cardio-facial syndrome. J Pediatr 2013;163:187-93.

[37] Vorstman JAS, Morcus MEJ, Duijff SN, et al. The 22q11.2 deletion in children: high rate of autistic disorders and early onset of psychotic symptoms. J Am Acad Child Adolesc Psychiatry 2006;45:1104-13.

[38] O'Hanlon JF, Ritchie RC, Smith EA, et al. Replacement of antipsychotic and antiepileptic medication by L-alpha methyldopa in a woman with velocardiofacial syndrome. Int Clin Psychopharmacol 2003;18:117-9.

[39] Graf WD, Unis AS, Yates CM, et al. Catecholamines in patients with 22q11.2 deletion syndrome and the low-activity COMT polymorphism. Neurology 2001;57:410-6.

[40] Mossaheb N, Schäfer MR, Schlögelhofer M, et al. Effect of omega- 3 fatty acids for indicated prevention of young patients at risk for psychosis: when do they begin to be effective? Schizophr Res 2013;148:163-7.
[41] Gothelf D, Gruber R, Presburger G, et al. Methyphenidate treatment for attention-deficit/hyperactivity disorder in children and adolescents with velocardiofacial syndrome: an open-label study. J Clin Psychiatry 2003;64:1163-9.

[42] Green T, Weinberger R, Diamond A, et al. The effect of methylphenidate on prefrontal cognitive functioning, inattention, and hyperactivity in velocardiofacial syndrome. J Child Adolesc Psychopharmacol 2011;21:589-95.

[43] Holmes J, Gathercole SE, Place M, et al. "Working memory deficits can be overcome: impacts of training and medication on working memory in children with ADHD' '. Appl Cognit Psychol 2010;24:827-36.

[44] Lohaugen GC, Gramstad A, Evensen KA, et al. Cognitive profile in young adults born preterm at very low birthweight. Dev Med Child Neurol 2010;52:1133-8.

[45] Demily C. Remédiation cognitive et microdélétion 22q11. In: Franck N, editor. Remédiation cognitive. Paris: Elsevier Masson; 2012. p. 240-8.

[46] Harrell W, Eack S, Hooper SR, et al. Feasibility and preliminary efficacy data from a computerized cognitive intervention in children with chromosome 22q11.2 deletion syndrome. Rev Dev Disabil 2013;34:2606-13.

[47] Glaser B, Lothe A, Chabloz M, et al. Candidate socioemotional program for individuals with intellectual disability. Am J Intellect Dev Disabil 2012;117:368-83. 